\title{
Dynamic Stall on High-Lift Airfoil 30P30N in Ground Proximity
}

\author{
Mohamed Sereez ${ }^{1 *}$, Umayr Zaffar ${ }^{2}$ \\ ${ }^{1}$ School of Engineering and Sustainable Development, De Montfort University, Leicester, UK \\ ${ }^{2}$ De Montfort University, Leicester, UK \\ Email: *mohamedshereez@hotmail.com
}

How to cite this paper: Sereez, M. and Zaffar, U. (2021) Dynamic Stall on High-Lift Airfoil 30P30N in Ground Proximity. Open Journal of Fluid Dynamics, 11, 135-152. https://doi.org/10.4236/ojfd.2021.113008

Received: March 19, 2021

Accepted: July 30, 2021

Published: August 2, 2021

Copyright () 2021 by author(s) and Scientific Research Publishing Inc. This work is licensed under the Creative Commons Attribution International License (CC BY 4.0).

http://creativecommons.org/licenses/by/4.0/

\begin{abstract}
Computational prediction of stall aerodynamics in free air and in close proximity to the ground considering the $30 \mathrm{P} 30 \mathrm{~N}$ three-element high-lift configuration is carried out based on CFD simulations using the OpenFOAM code and Fluent software. Both the attached and separated flow regimes are simulated using the Reynolds Averaged Navier-Stokes (RANS) equations closed with the Spalart-Allamaras (SA) turbulence model for static conditions and pitch oscillations at Reynolds number, $R e=5 \times 10^{6}$ and Mach number, $M=0.2$. The effects of closeness to the ground and dynamic stall are investigated and the reduction in the lift force in close proximity to the ground is discussed.
\end{abstract}

\section{Keywords}

Dynamic Stall, High-Lift Airfoil, Ground Effect

\section{Introduction}

High-lift wing configuration systems are optimized for takeoff and landing, so the effect of proximity to the ground on their aerodynamics is an important part of the design process [1] [2] [3]. Loss of control in flight (LOC-I) due to aerodynamic stall is most critical in close proximity to the ground due to lack of recovery time from aircraft stall upsets [4] [5]. From another perspective, airframe noise reduction during approach and landing is currently an important problem in aviation and therefore computational prediction of the level of noise for a high lift wing with a deflected slat is now an active research area [6]. Additionally, a better understanding of flow separation processes on high-lift wing configurations in close proximity to the ground is essential for the development of adequate flight simulation models for pilot training and improvement of flight 
safety [7].

Computational analysis of the flow around two-dimensional airfoils and low aspect ratio wing configurations underground effect conditions has been the focus of attention in the literature [8] [9] [10] [11]. For example, the impact of ground on the aerodynamic performance and stability of the CRM-WB-HVT (Common Research Model-Wing-Body-Horizontal-Vertical Tail) aircraft configuration in both the longitudinal and lateral directions, was studied in [12]. Most of the computational and experimental studies have been focused either on predicting the transition to flow separation regimes or on estimating the level of noise [1] [6].

Investigation of aerodynamic stall and dynamic stall hysteresis phenomenon in free air for various flow conditions with a broad range of Reynolds numbers has attracted significant attention in the literature [13]-[23], partly from the perspective of the development of Reduced Order Models (ROM) based on phenomenological principles [17] [24] [25] [26]. However, there is a lack of studies in stall aerodynamics taking into account the ground effect. The motivation of our study lies in the extension of dynamic stall hysteresis simulation in close proximity to the ground, considering a three-element high-lift 30P30N airfoil.

In this paper, the study focuses on the aerodynamics of the 30P30N high-lift airfoil system in close proximity to the ground including the post-stall region in both static and dynamic conditions. The computational prediction of aerodynamic characteristics of the $30 \mathrm{P} 30 \mathrm{~N}$ high-lift airfoil system at a moderately high Reynolds number of $R e=5 \times 10^{6}$ and Mach number $M=0.2$ was performed using the OpenFOAM code [27] and Fluent software [28] in the angle of attack range covering the stall zone. The experimental results for the $30 \mathrm{P} 30 \mathrm{~N}$ airfoil [2] are used for validation of the obtained simulation results. The Unsteady Reynolds Averaged Navier-Stokes (URANS) equations in both OpenFOAM and Fluent were solved with the use of the Spalart-Allmaras (SA), one-equation eddy viscosity model [29].

The paper is organized as follows. The computational framework including the mesh generation and blocking techniques, time integration methodology, and numerical setup is described in Section 2. The obtained CFD simulation results for fixed angles of attack and oscillatory-pitching motion together with flow visualizations are presented in Section 3. The concluding remarks and plans for the future work are presented in Section 4.

\section{Computational Framework}

The section describes the $30 \mathrm{P} 30 \mathrm{~N}$ airfoil geometry, grid generation techniques, URANS equations, turbulence modelling, and the numerical setup which was employed in all simulations in this study. Identical numerical setups were used for the OpenFOAM code and the Fluent software during CFD simulations for consistency of the obtained results.

\subsection{Geometry and Grid Generation}

The high-lift airfoil 30P30N shown in Figure 1 is comprised of a slat, main air- 
foil and the flap section. The geometrical features of this airfoil are shown in Table 1, where $c$ is the stowed chord, $\delta_{s}$ and $\delta_{f}$ are the deflection angle of the slat and flap section and $g_{s}$ and $g_{f}$ are the gap for the slat and flap. The grids for the $30 \mathrm{P} 30 \mathrm{~N}$ geometry are generated using a structured blocking approach, with two fluid zones connected by an Arbitrary Mesh Interface (AMI). The AMI with two fluid zones helps to further extend the simulations to dynamic loops in both free air and in close proximity to the ground. The structured blocking approach for the high-lift 30P30N airfoil was utilized such that the cell skewness and the cell non-orthogonality are maintained at good quality in order to avoid divergence and improve the convergence process in the URANS solver. The far field extends 50 chord lengths away from the high lift airfoil in all directions. The cell size transition from the far field of the domain to the airfoil is small enough to avoid large variation of gradients of vector and scalar fields. Furthermore, the gaps between the slat, main airfoil and flap are well refined in order to accurately capture any transition or separation, which is often the case for highlift configuration airfoils. The boundary layers were modelled with an O-type blocking with dimensionless normal wall distance $Y+$ less than unity i.e., $Y+\leq 1$, consisting 40 layers growing in the normal direction with a factor of 1.08. A close-up view of the resulting mesh with 267,392 grid points and 131,856 quadrilateral elements is shown in Figure 2.

The boundary conditions used in the OpenFOAM and Fluent are defined as $U_{\text {wall }}=0, \hat{v}_{\text {wall }}=0, \hat{v}_{\text {farfield }}=3 v_{t \infty}$ to $5 v_{t \infty}$, and $v_{t \text { wall }} \approx 0$ and $\partial p /\left.\partial x\right|_{\text {wall,farfield }}=0$, where $U$ is the velocity, $v_{t}$ is the turbulent viscosity, $\hat{v}$ is the modified turbulent viscosity, and $\partial p / \partial x$ is the pressure gradient. The boundary condition on the ground is implemented using a "moving WallBoundary" function in both static and pitching motion cases.

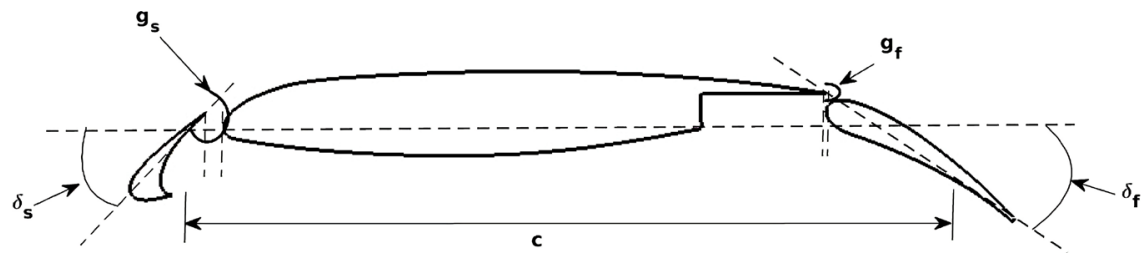

Figure 1. Geometrical aspects of the high-lift 30P30N airfoil.

Table 1. Geometrical aspects of 30P30N airfoil.

\begin{tabular}{ccccc}
\hline$c$ & $\delta_{s}$ & $\delta_{f}$ & $g_{s}$ & $g_{f}$ \\
\hline $0.457 \mathrm{~m}$ & $30^{\circ}$ & $30^{\circ}$ & $2.95 \%$ & $1.27 \%$ \\
\hline
\end{tabular}

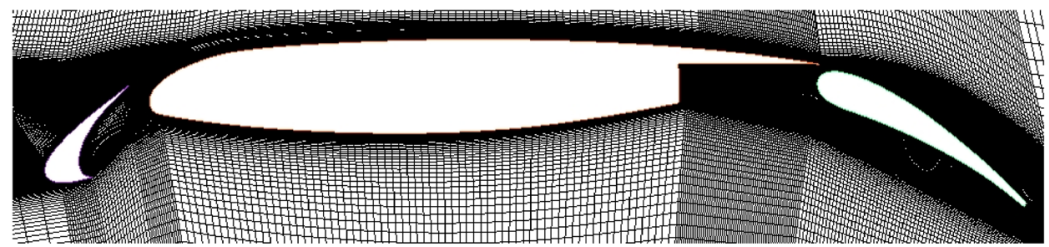

Figure 2. Structured grid adopted for the $30 \mathrm{P} 30 \mathrm{~N}$ high-lift airfoil at $R e=5.0 \times 10^{6}$. 


\subsection{Governing Equations}

The Navier-Stokes (NS) equations for the case of incompressible flow include the continuity equation:

$$
\nabla \cdot \boldsymbol{u}=0
$$

and the momentum equation:

$$
\frac{\partial \boldsymbol{u}}{\partial t}+(\boldsymbol{u} \cdot \nabla) \boldsymbol{u}-v \nabla^{2} \boldsymbol{u}=-\frac{\nabla \boldsymbol{p}}{\rho}
$$

The computational resources required for direct numerical simulations (DNS) of Equations (1) and (2) usually exceed currently available capabilities. The effect of turbulence is normally simplified by solving the unsteady Reynolds-averaged Navier-Stokes (URANS) equations, which are a time-averaged approximation of the NS equations. The averaging of fluctuating velocities generates additional terms, known as the Reynolds stresses. To describe these stresses, additional empirical equations, either algebraic or differential, are required to close the computational system. The majority of URANS turbulence models are based on the concept of eddy viscosity, equivalent to the kinematic viscosity of the fluid and describing the turbulent mixing or diffusion of the flow momentum [27]. The Reynolds stresses appearing in the URANS equations due to averaging, are normally modelled in the linear approximation with the well known Boussinesq assumption as shown in Equation (3):

$$
\tau_{i j}=2 \mu_{t}\left(S_{i j}-\frac{1}{3} \frac{\partial u_{k}}{\partial x_{k}} \delta_{i j}\right)-\frac{2}{3} \rho k \delta_{i j}
$$

\subsection{Turbulence Model}

The SA [29], one equation turbulence model, which is commonly used in aeronautical applications and specifically developed for external aerodynamics involving adverse pressure gradients and strongly separated flow conditions [17] is employed in this study. Furthermore, the SA model is computationally cheaper than the Shear-Stress Transport (SST) model [30] which is also widely used for flows with strong separation conditions. The SA model solves for the modified turbulent viscosity $\hat{v}$ and is described by the partial differential equation in Equation (4).

$$
\begin{aligned}
\frac{\partial \hat{v}}{\partial t}+u_{j} \frac{\partial \hat{v}}{\partial x_{j}}= & C_{b 1}\left(1-f_{t 2}\right) \hat{S} \hat{v}-\left(C_{w 1} f_{w}-\frac{C_{b 1} f_{t 2}}{\kappa^{2}}\right) \frac{\hat{v}}{d^{2}} \\
& +\frac{1}{\sigma_{t}}\left[\frac{\partial}{\partial x_{j}}(v+\hat{v}) \frac{\partial \hat{v}}{\partial x_{j}}+C_{b 2} \frac{\partial \hat{v}}{\partial x_{i}} \frac{\partial \hat{v}}{\partial x_{i}}\right]
\end{aligned}
$$

The turbulent viscosity is then obtained from

$$
v_{t}=\rho \hat{v} f_{v 1}
$$

where

$$
f_{v 1}=\frac{X^{3}}{X^{3}+C_{v 1}^{3}}
$$


and

$$
X=\frac{\hat{v}}{v}
$$

The SA model constants are given in Table 2. In Equation (4), the production of turbulence is described by the term

$$
C_{b 1}\left(1-f_{t 2}\right) \hat{S} \hat{v}
$$

the diffusion of turbulence is described by the term

$$
\frac{1}{\sigma_{t}}\left[\frac{\partial}{\partial x_{j}}(v+\hat{v}) \frac{\partial \hat{v}}{\partial x_{j}}+C_{b 2} \frac{\partial \hat{v}}{\partial x_{i}} \frac{\partial \hat{v}}{\partial x_{i}}\right]
$$

and the destruction of turbulence is described by the term

$$
\left(C_{w 1} f_{w}-\frac{C_{b 1} f_{t 2}}{\kappa^{2}}\right) \frac{\hat{v}}{d^{2}}
$$

The Dual Time Stepping (DTS) method is used to simulate both the static and the dynamic loops and the DTS can be described by set of equations as follows [27] [31].

$$
A+=\left(\frac{3}{2 \Delta t}+\frac{1}{\alpha_{k} \Delta \tau}\right) \int_{S} \mathrm{~d} S \mathrm{~d} A
$$

which

$$
H^{\prime}+=\left(\left(\frac{1}{\alpha_{k} \Delta \tau}\right) U^{m-1}+\left(\frac{2}{\Delta t}\right) U^{n-1}-\left(\frac{1}{2 \Delta t}\right) U^{n-2}\right) \int_{S} \mathrm{~d} S \mathrm{~d} A
$$

where is $\mathrm{n}$ is the time step level, $\mathrm{m}$ is the sub-iteration level within each time step, $\alpha_{k}$ is the Runge-Kutta coefficient in each stage and $\int_{S} \mathrm{~d} S \mathrm{~d} A$ is the integrated volume of each element. The term $\Delta \tau$ is obtained by using either a) the Stabilized Local Time Stepping Scheme (SLTS) or the Courant Number Limited Euler Method (CoEuler) Scheme, which are already implemented in OpenFOAM. The SLTS scheme guarantees a locally stabilized time step size so that the contribution to FVM matrix due to advective/convective terms remain diagonally dominant and the CoEuler scheme maintains the time step size such that the local flow courant number does not exceed the specified values by the user.

The use of a dual time stepping method with 30 - 50 iterations in the inner loop ensures that the continuity equation is fully satisfied, such that the residuals of the flow field at every time step are dropped at least 5 magnitudes of order, which seemed to be sufficient for this study. Furthermore, with the use of the Courant number based local time stepping technique (CoEuler), the local CFL number was kept below 5, enabling accurate capture of the time scale of any eddies that may be formed during static or dynamic hysteresis conditions.

Table 2. SA turbulence model coefficients.

\begin{tabular}{ccccccccc}
\hline$\sigma_{t}$ & $C_{b 1}$ & $C_{b 2}$ & $\kappa$ & $C_{w 1}$ & $C_{w 2}$ & $C_{w 3}$ & $C_{v 1}$ & $C_{t 1}$ \\
\hline $2 / 3$ & 0.1355 & 0.622 & 0.41 & $C_{b 1} / \kappa^{2}+\left(1+C_{b 2}\right) / \sigma_{t}$ & 0.3 & 2 & 7.1 & 1 \\
\hline
\end{tabular}




\section{Results}

The 30P30N airfoil is first simulated in static conditions with no mesh motion and fixed angle of attack, $\alpha$, using URANS solver and with varying height to chord ratio $(h / c)$ from the ground. The "free-air" condition is described as far away from the ground $(h / c=\infty)$, such that there is no influence of the ground on the pressure generated either on the suction or pressure side of the $30 \mathrm{P} 30 \mathrm{~N}$ airfoil. The $h / c$ parameter is a dimensionless parameter characterising closeness to the ground. A summary of the investigated cases is presented in Table 3 .

Once the analysis of the ground effect characteristics of the $30 \mathrm{P} 30 \mathrm{~N}$ airfoil in static conditions was completed, the airfoil was then simulated in oscillating pitching motion i.e. dynamic loops with specific reduced frequency, $k$, where $k=\omega c / 2 U_{r e f}$ in a) free-air conditions $(h / c=\infty)$ and b) at $h / c=0.8$. A summary of the setup for dynamic mesh motion cases is shown in Table 4. In Table 4, $\omega$ is angular rotation rate in $\mathrm{rad} / \mathrm{s}$ and $c$ is the stowed chord length in metres.

\subsection{Validation of CFD Results}

This subsection presents the computational results validation using a) comparison of pressure coefficient, $C_{P}$, against experimental results from [2]; and b) the lift force convergence study for three fixed angle of attacks i.e. $\left(\alpha=0,4\right.$ and $\left.8^{\circ}\right)$. The validation is carried out at static conditions with no mesh motion and the Reynolds number, $R e=5.0 \times 10^{6}$ with respect to airfoil stowed chord length and Mach number, $M=0.2$.

The pressure coefficient, $C_{P}$ distribution obtained in OpenFOAM at angle of attack, $\alpha=8.0^{\circ}$, is shown in Figure 3. There is an excellent agreement of pressure coefficient variation over the slat, main airfoil section and the flaps. The maximum suction pressure is observed at the very start of the leading edge of the main airfoil with $C_{P} \approx-6.0$ as shown by experimental results from Klausmeyer [2]. This gives us confidence to move forward with other test cases as planned using the currently adopted structured grid generated for the $30 \mathrm{P} 30 \mathrm{~N}$ airfoil.

Table 3. Summary of case setup in static conditions with fixed angle of attack, $\alpha$, for the 30P30N airfoil.

\begin{tabular}{cccccc}
\hline case & $h / c$ & $U_{r e f}(\mathrm{~m} / \mathrm{s})$ & $R e$ & range of $\alpha$, degrees & CFD code \\
\hline 1a & $\infty$ & 68.6 & $5.0 \times 10^{6}$ & $0-26,0-12$ & OpenFOAM, Fluent \\
1b & 1.0 & 68.6 & $5.0 \times 10^{6}$ & $0-12$ & Fluent \\
1c & 0.8 & 68.6 & $5.0 \times 10^{6}$ & $0-24$ & OpenFOAM \\
\hline
\end{tabular}

Table 4. Summary of OpenFOAM case setup for dynamic loops with $\alpha(t)=\alpha_{m}+\alpha_{a} \sin (\omega t)$ for the $30 \mathrm{P} 30 \mathrm{~N}$ airfoil.

\begin{tabular}{cccccc}
\hline case & $h / c$ & $U_{\text {ref }}(\mathrm{m} / \mathrm{s})$ & $R e$ & frequency $(\mathrm{Hz})$ & reduced frequency $\left(k=\omega c / 2 U_{\text {ref }}\right)$ \\
\hline $2 \mathrm{a}$ & $\infty$ & 68.6 & $5 \times 10^{6}$ & $0.5,1.0,1.5$ & $0.010,0.021,0.031$ \\
$2 \mathrm{~b}$ & 0.8 & 68.6 & $5 \times 10^{6}$ & $1.0,1.5$ & $0.021,0.031$ \\
\hline
\end{tabular}




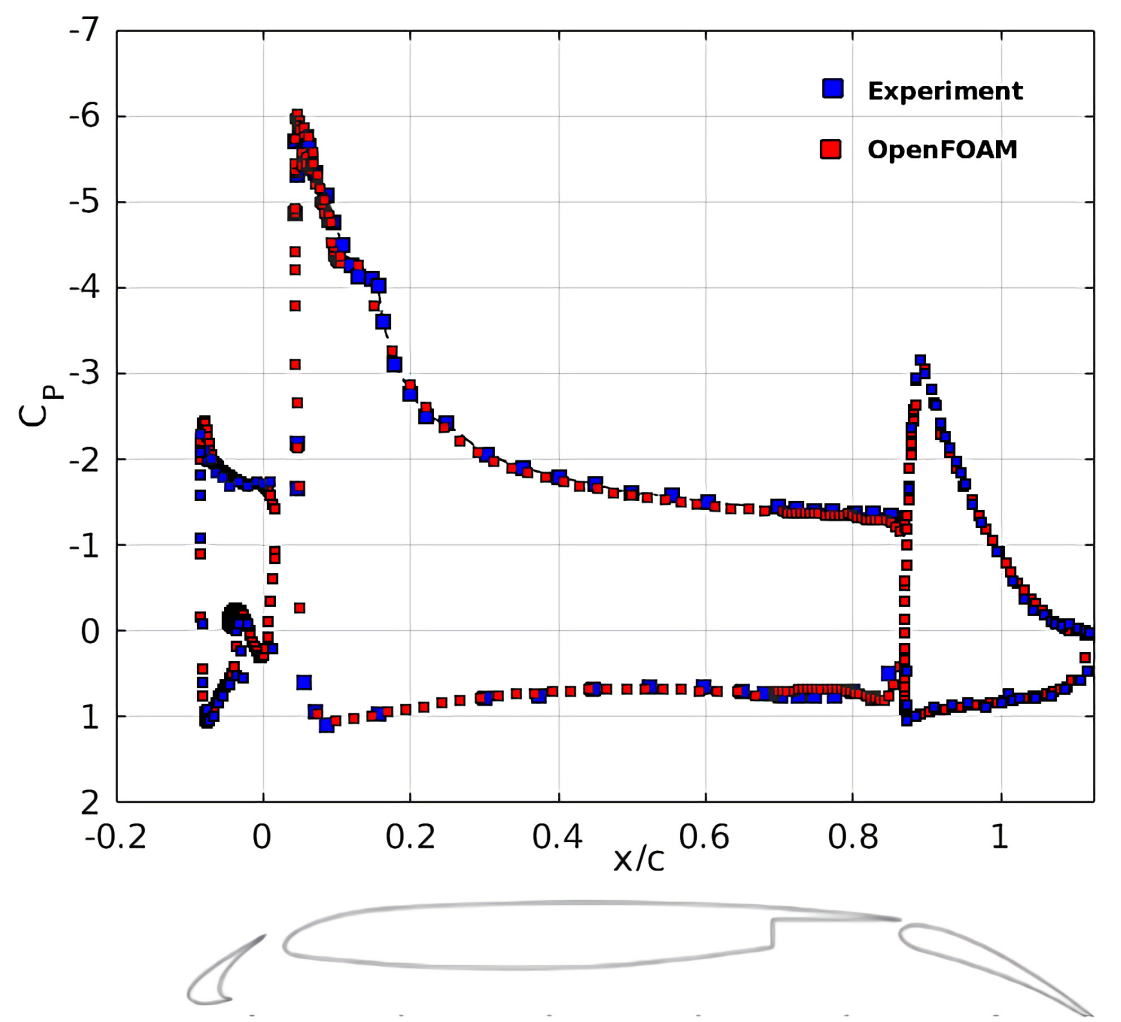

Figure 3. Validation of pressure coefficient, $C_{P}$ distribution for $30 \mathrm{P} 30 \mathrm{~N}$ airfoil at $R e=5 \times 10^{6}$ and experimental results from [16].

The obtained results for the force convergence study are shown in Figure 4. The lift coefficient converges to constant values at flow time of $t=1.0 \mathrm{~s}$ for all the considered angle of attacks.

\subsection{Simulation of the Static Stall in Flow past 30P30N Airfoil}

The static variation of angle of attack for flow past high-lift $30 \mathrm{P} 30 \mathrm{~N}$ is carried out at static conditions with no grid motion and covering attached and separated flow regions. The simulations are performed for in and out of ground effect, using two different CFD codes OpenFOAM and Fluent. The obtained results for the lift coefficients for the considered cases ( $1 \mathrm{a}, 1 \mathrm{~b}$ and $1 \mathrm{c}$ ) are shown in Figure 5. The $h / c=\infty$ in Figure 5 simply means that the airfoil is far away from the ground such that no influence of ground/runway is on the aerodynamic characteristics of the airfoil.

The results for the clean configuration with $h / c=\infty$ from Figure 5, show attached flow conditions with linear variation of the lift force coefficient $C_{L}$ up to angle of attack, $\alpha=15^{\circ}$. From 15 degrees of angle of attack, the $C_{L_{\alpha}}$ slope starts to decline, indicating increasing separation over the airfoil. The $C_{L_{\max }}$ is at $\alpha=22^{\circ}$ which is the same as obtained in the experimental results shown in [1]. At $\alpha=26^{\circ}$ a sharp drop in the lift force coefficient is observed. This happens as the flow separation has reached full development with indication of a significantly strong stall for the 30P30N high-lift airfoil. 


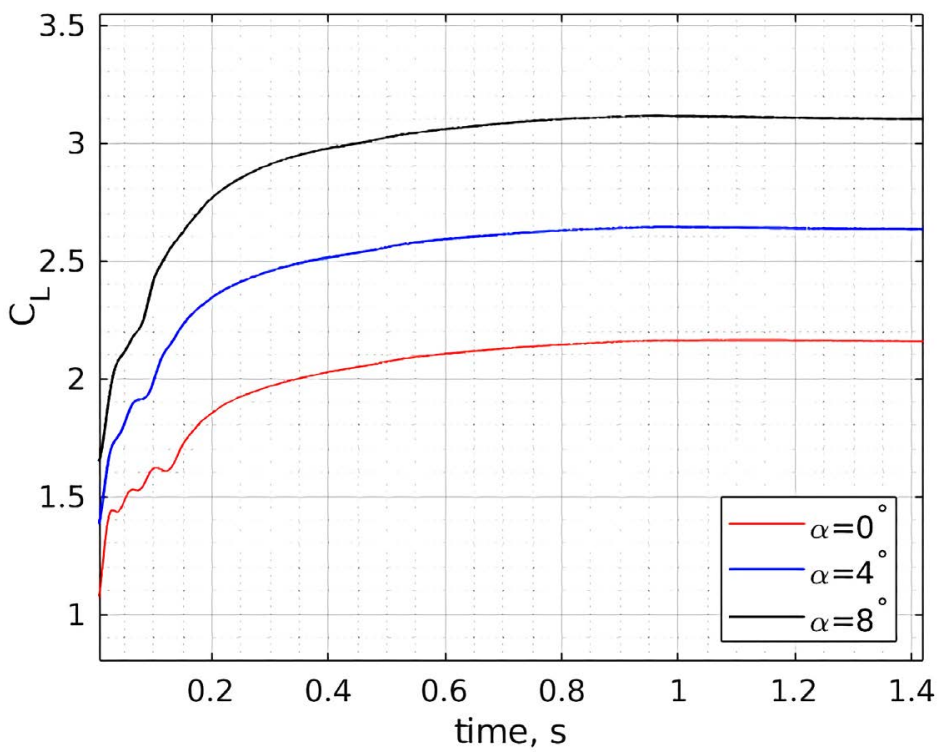

Figure 4. Force convergence study of the lift coefficient, $C_{L}$ for $30 \mathrm{P} 30 \mathrm{~N}$ airfoil at $R e=5 \times 10^{6}$.

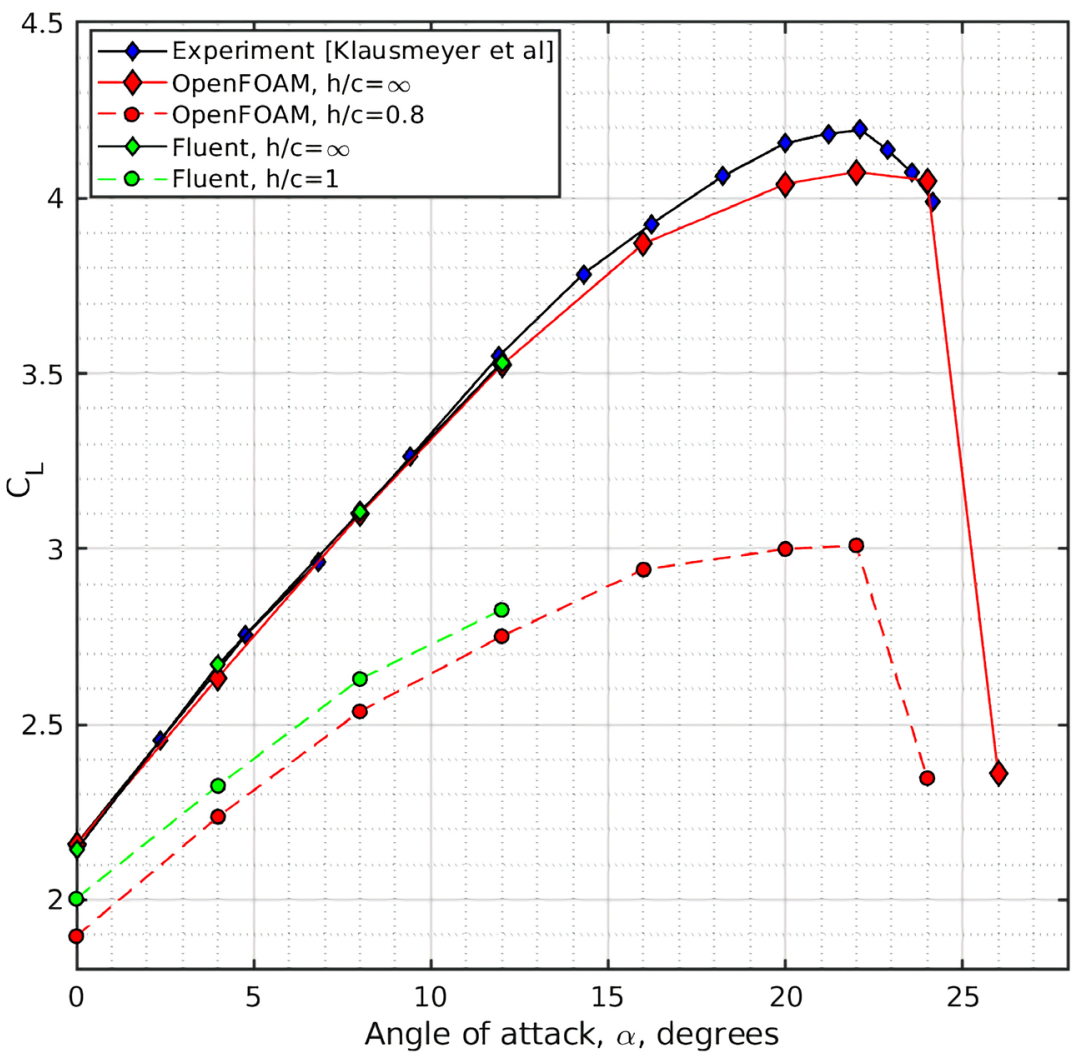

Figure 5. Lift force coefficient, $C_{L}$, for various $h / c$ ratios at $R e=5 \times 10^{6}$ and experimental results from [16].

The results for the $30 \mathrm{P} 30 \mathrm{~N}$ airfoil in ground effect with close proximity to the ground, shows a considerably large degradation in the aerodynamic characteristics. Both the OpenFOAM simulations at $h / c=0.8$ and Fluent simulations at 
$h / c=1.0$ show a large drop in the lift force coefficient values in comparison to the "clean" airfoil with $h / c=\infty$. The results from OpenFOAM code at $h / c=0.8$ in Figure 5 (see red dashed line with circle markers) show that the reduction in the lift coefficient $C_{L}$ values is bigger in comparison to the $C_{L}$ values obtained for fluent simulations at $h / c=1.0$. For the ground effect case simulated in OpenFOAM with $h / c=0.8$, maximum lift coefficient, $C_{L_{\max }}$, is observed at $\alpha=22^{\circ}$ with a value of $C_{L_{\max }} \approx 3.0$ which is almost a $27 \%$ reduction in the maximum lift coefficient.

Most of the computational results reported in literature demonstrated that with the decrease of the distance between the airfoil and the ground, typically denoted by the vertical distance of airfoil's center of moment from ground to the airfoil chord ratio i.e., $(h / c)$, an increase in the lift coefficient, $C_{L}$ values are shown [8] [12]. However, for the high-lift configurations there have been noticeable deviations in this trend, showing a decrease of the lift coefficient, $C_{L}$ as the airfoil approaches the ground/runway. Such computational case studies with significant reduction in the lift force were reported in [32] [33]. The DATCOM (Section 4.7) [3], presents results showing that a transport aircraft with a slotted flap wing show a noticeable reduction in the lift force in close proximity to the ground. Paper [34] reports similar kind of decrease of the lift force coefficient, $C_{L}$ in close proximity to the ground, where the pressure loss in the suction side was much bigger the increment of pressure caused due to increased closeness to the ground.

A noticeable reduction in the lift force coefficient values is shown in the work by Parth [35], where a modified discrete vortex method-based model along with the Computational Fluid Dynamics (CFD) was used for the prediction of 2D/3D ground effect for various high-lift airfoil and wing configurations. Within their simulation of $30 \mathrm{P} 30 \mathrm{~N}$ airfoil, for the angle of attack of $\alpha=8.12^{\circ}$, at flow conditions of Reynolds number, $R e=5 \times 10^{6}$ and $M=0.2$, the drop in the lift coefficient $C_{L}$ between $h / c=20$ and $h / c \leq 0.4$ was approximately equal to a significant $\Delta C_{L} \approx 1.03$. This large drop of the lift coefficient by a factor of roughly 1.47 should be kept in consideration, as significant research is now focused on lift and drag force coefficient optimization for high-lift airfoils. To be more specific, the lift and drag optimizations such as found in [36] should be simulated with consideration of how high-lift airfoils behave in ground effect with close proximity to the runway.

A comparison of the pressure coefficient, $C_{P}$ distribution, for the slat section, main airfoil and the flap section at three different angle of attacks i.e. $\alpha=0,4$ and $8^{\circ}$ are shown in Figure 6 As the angle of attack increases the maximum negative pressure generated on the upper surface of the main airfoil increases significantly. However, with the increase of angle of attack, the $C_{P}$ distribution on the flaps do not change by a large extent, while at $\alpha=8^{\circ}$ the pressure distribution on the slats increase significantly.

Figure 7 shows the difference in pressure coefficient distribution in and out of 


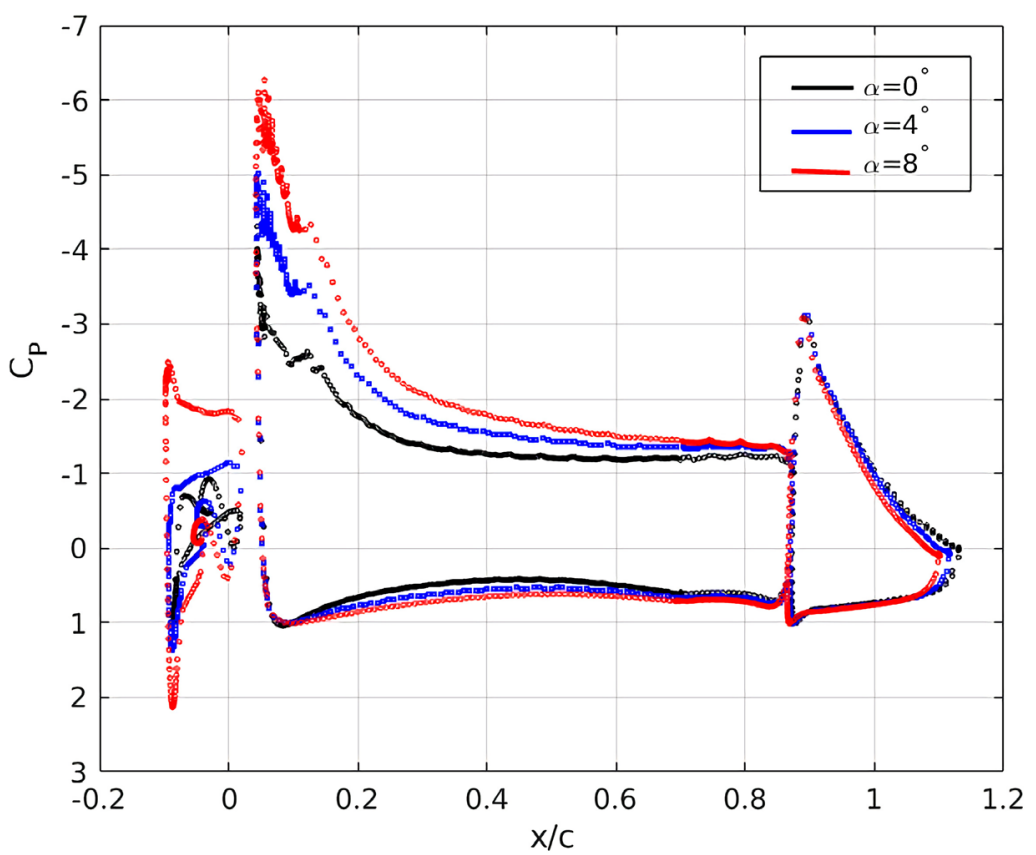

Figure 6. Comparison of pressure coefficient, $C_{P}$, distribution at $h / c=\infty$ for various angle of attack.

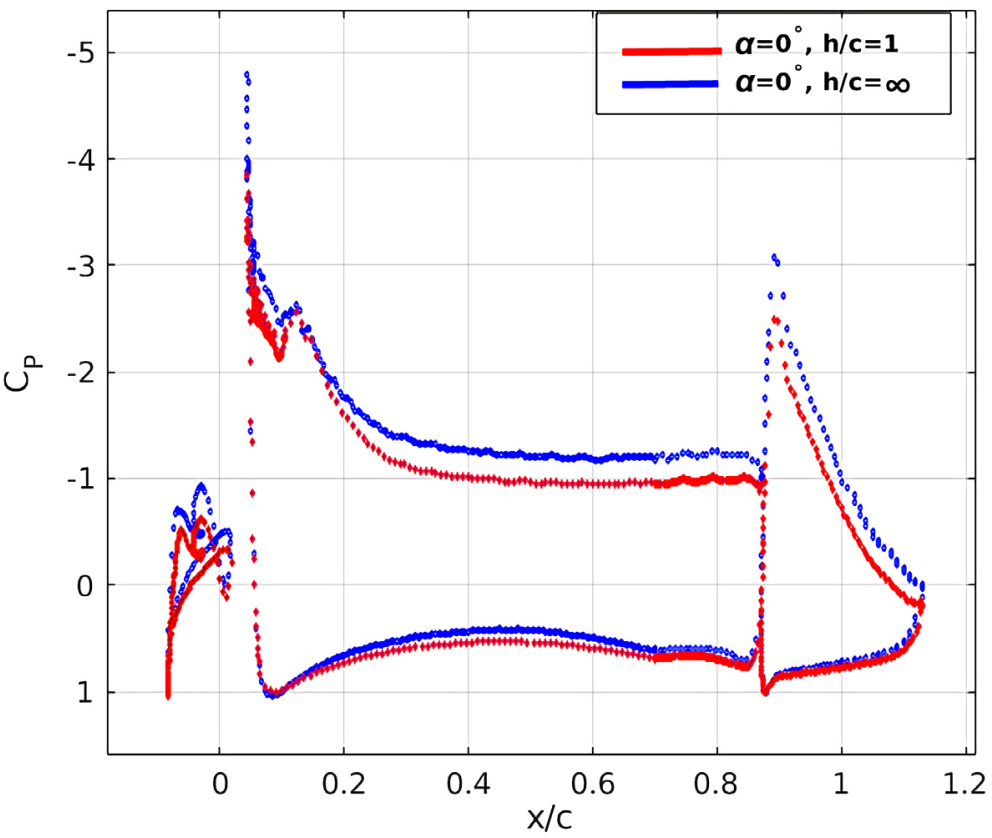

Figure 7. Comparison of pressure coefficient, $C_{P}$ distribution at $h / c=\infty$ and $h / c=1.0$ for angle of attack, $\alpha=0^{\circ}$.

ground effect for the $30 \mathrm{P} 30 \mathrm{~N}$ airfoil. It is evident that there is a slight increase in the integral of the pressure developed on the lower side/pressure side of the airfoil. However, in comparison to the free air simulations with $h / c=\infty$, the main airfoil loses significant pressure at the leading edge when $h / c=1$. Additionally, it can be noticed that the amount of pressure generated in the flap section of the 
airfoil is reduced for the considered angle of attack of $\alpha=0^{\circ}$.

To understand the effect of ground on the aerodynamic characteristics of the airfoil, the skin friction coefficient, $C_{f}$ is analyzed for the $30 \mathrm{P} 30 \mathrm{~N}$ airfoil and the results are shown in Figure 8. The higher skin friction coefficient for the clean airfoil in Figure 8 (blue circles) indicates more attached flow conditions in comparison to the ground effect case with $h / c=1$ (see red circles), where a lower skin friction coefficient was observed. A lower skin friction coefficient usually indicates separated flow regions leading to lower lift coefficient values as shown in Figure 5.

\subsection{Simulation of Dynamic Stall for Flow past 30P30N Airfoil}

In this section, the results for the dynamic loops in and out of ground effect for the $30 \mathrm{P} 30 \mathrm{~N}$ airfoil are presented. The dynamic loops are carried out at three different frequencies of $0.5,1$ and $1.5 \mathrm{~Hz}$ (reduced frequencies of $k=0.010,0.021$ and 0.031 ). The DTS method along with a local time stepping for the pseudo time integration is used for time advancement. The cell limited least squares evaluation was employed for flow vector/scalar gradients and second order accuracy for all flow quantities such as velocity and turbulence were maintained. The inner fluid zone made of a smaller circle domain inside the viscous fluid outer domain rotates using an angular oscillatory rotation function, while the outer fluid zone is kept in static non-moving conditions. The mean angle of attack, $\alpha_{m}$ for the dynamic loops is $\alpha_{m}=16^{\circ}$ and the amplitude of the pitch angle is $\alpha_{a}=15.0^{\circ}$. The frequencies $f=0.5,1.0$ and $1.5 \mathrm{~Hz}$ are representative of angular rotation rates often used in wind tunnel tests for estimation of aerodynamic derivatives of aircraft. Large amplitude oscillations with amplitude of

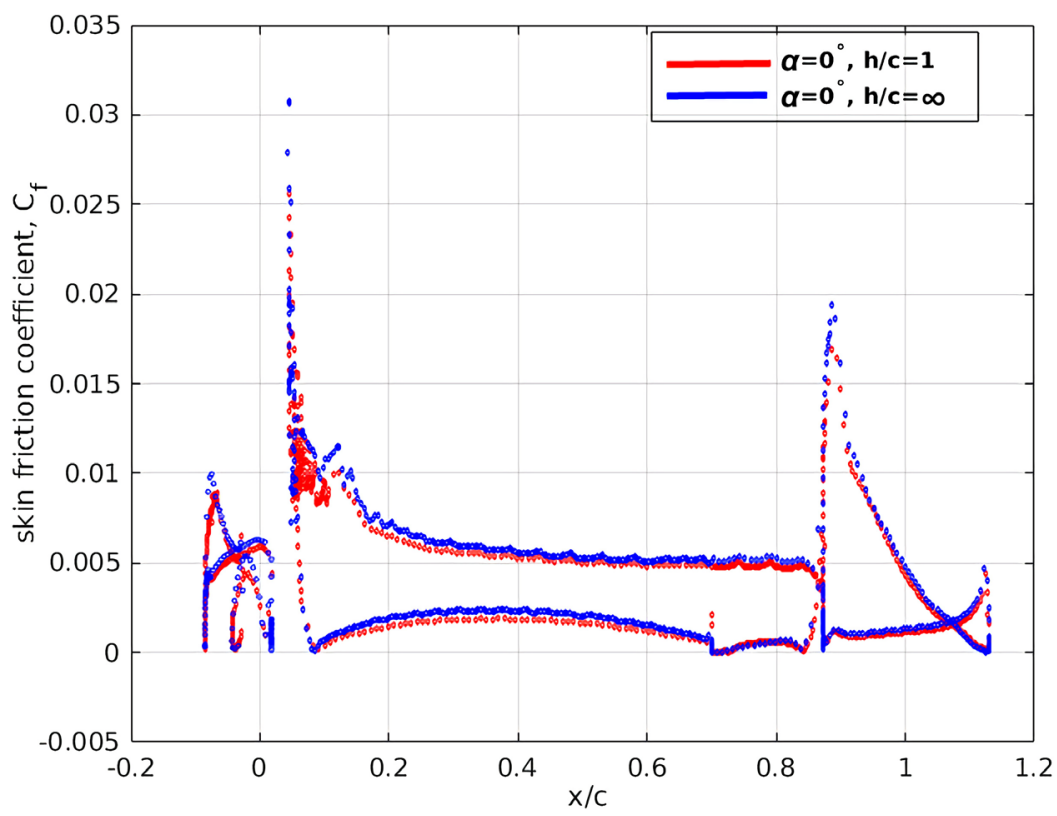

Figure 8. Comparison of skin friction coefficient, $C_{f}$ distribution in clean conditions vs ground effect at $\alpha=0^{\circ}$. 
$\alpha_{a}=15.0^{\circ}$ was chosen in order to cover the stall region and evaluate the dynamic stall effects on the $30 \mathrm{P} 30 \mathrm{~N}$ airfoil in close proximity to the ground. The dynamic loops for variation of the aerodynamic coefficients are repeated for 3 cycles and the data from the final cycle have been extracted in order to eliminate transients in the flow dynamics. The angle of attack variation in time is described by Equation (13)

$$
\alpha(t)=\alpha_{m}+\alpha_{a} \sin (\omega t)
$$

The dynamic loops obtained in OpenFOAM for the free air simulations with $h / c=\infty$ are illustrated in Figure 9. The obtained results indicate a significant increase in the $C_{L_{\max }}$ and a large delay in stall angle $\alpha_{s}$ for all the considered cases. When reduced frequency of $k=0.01$, the stall is delayed until $\alpha_{s} \approx 28^{\circ}$. This is followed by a significant drop in the lift coefficient (see Figure 9 magenta line). The re-attachment of $C_{L}$ does not occur until $\alpha=5^{\circ}$.

When the reduced frequency is increased to $k=0.021$, the stall angle $\alpha_{s}$ is further delayed to $\alpha_{s}=30^{\circ}$, with a maximum lift coefficient of $C_{L_{\max }} \approx 4.45$ (see Figure 9 blue line). The return branch is compromised of high-frequency vortex shedding and shows no re-attachment of flow back to static conditions until $\alpha=1^{\circ}$. When the reduced frequency is further increased to $k=0.031$ the return branch shows an even large drop in the lift coefficient. For instance at angle of attack, $\alpha=10^{\circ}$, the lift coefficient, $C_{L} \approx 1.7$, whereas the in the top branch the lift coefficient was a much higher value of $C_{L} \approx 3.2$.

The dynamic hysteresis observed for all the three reduced frequencies shows a

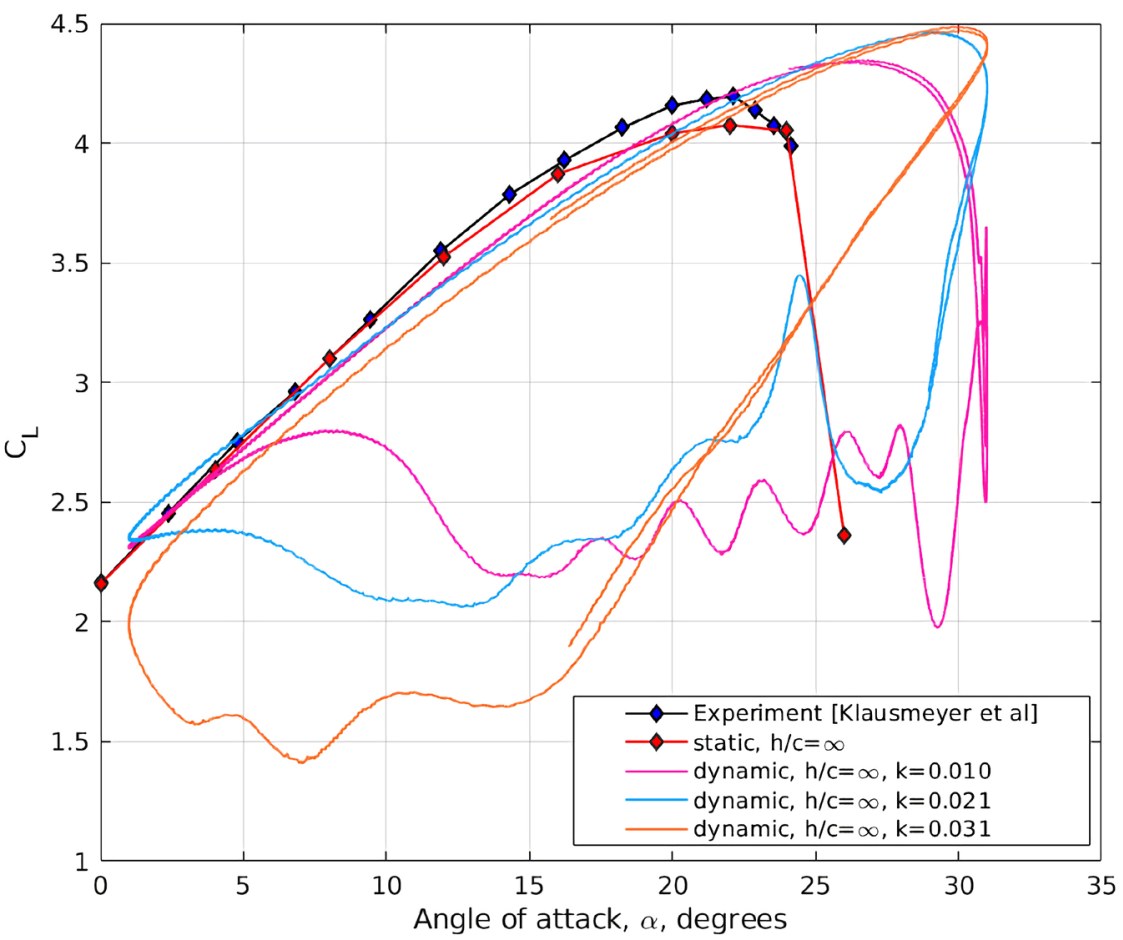

Figure 9. Lift force coefficient, $C_{L}$, for dynamic stall loops at Reynolds number, $R e=5 \times 10^{6}$, for 30P30N airfoil and $h / c=\infty$. 
large difference in the $C_{L}$ values between the upstroke and downstroke motion with no indication of reattachment for the entire duration of the return loop.

The dynamic hysteresis loops obtained for the cases with close proximity to the ground are shown in Figure 10. Two frequencies of $F=1.0$ and $1.5 \mathrm{~Hz}$ are used as the angular rotation rate. The results from Figure 10 show similar characteristics as shown in Figure 9. The dynamic loops lead to a large delay in the stall angle with an increase in $C_{L_{\max }}$ coefficient for the top branch. For the bottom branch the lift coefficients are significantly low and do not show return to static conditions until the angle of attack is equal to $\alpha=1^{\circ}$.

A comparison of the obtained dynamic hysteresis loops for free air and in close proximity to the ground is presented in Figure 11. In comparison to the free air simulations (see solid lines in Figure 11), the dynamic hysteresis loops obtained in ground proximity (see dashed lines in Figure 11) indicates lower lift coefficient $C_{L}$ values for the entire range of angle of attack variation.

The flow visualization for $\alpha=20^{\circ}$ and frequency, $f=1.0 \mathrm{~Hz}$ is shown in Figure 12. The top branch shows almost fully attached flow conditions while the bottom branch shows large separation regions above the main airfoil section which corresponds well to the $C_{L}$ values for the dynamic hysteresis loops shown in Figure 9. The contours for turbulent viscosity at two different angle of attacks $\alpha=11$ and $18^{\circ}$ in Figure 13, show no eddies or large separation regions during the upstroke, while during the downstroke motion for the same angle of attack, turbulent eddies are shed from the airfoil.

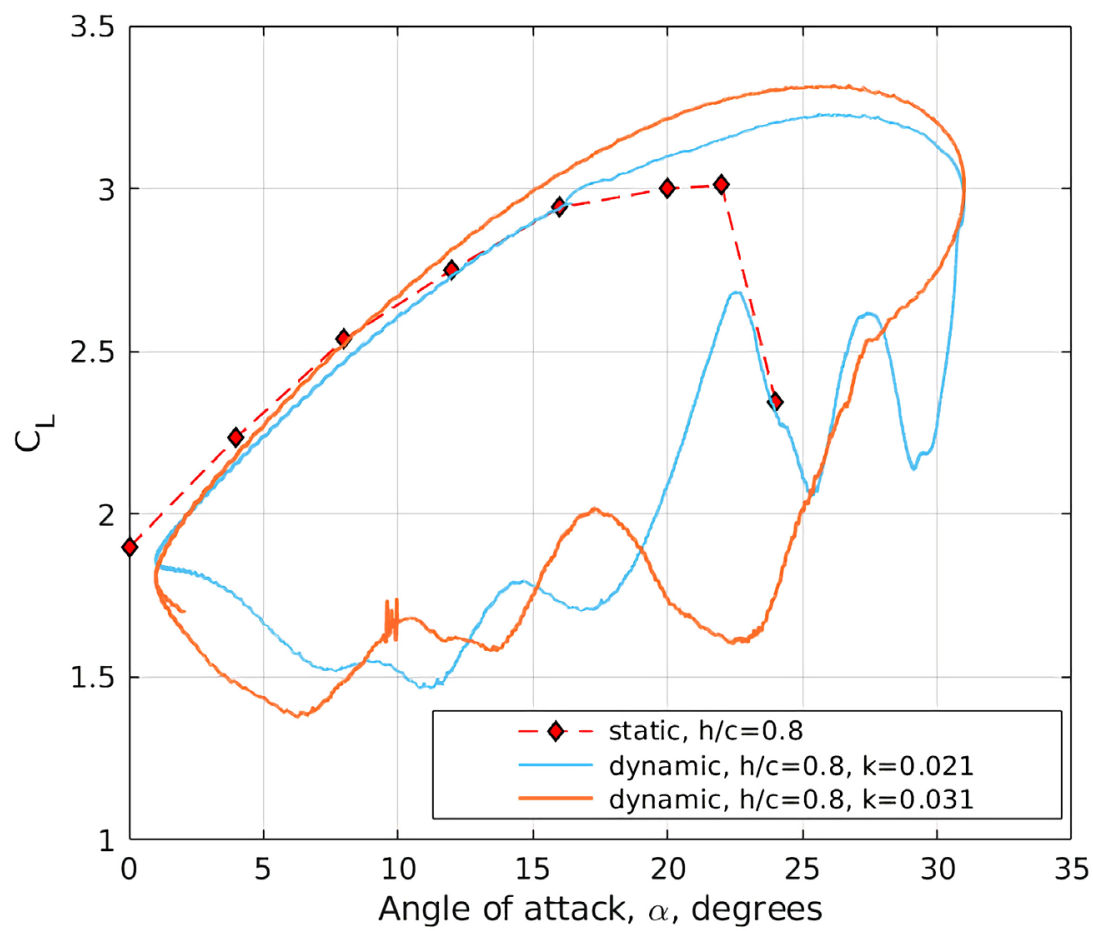

Figure 10. Lift force coefficient, $C_{L}$, for dynamic stall loops at Reynolds number, $R e=5 \times 10^{6}$, for $30 \mathrm{P} 30 \mathrm{~N}$ airfoil and $h / c=0.8$. 


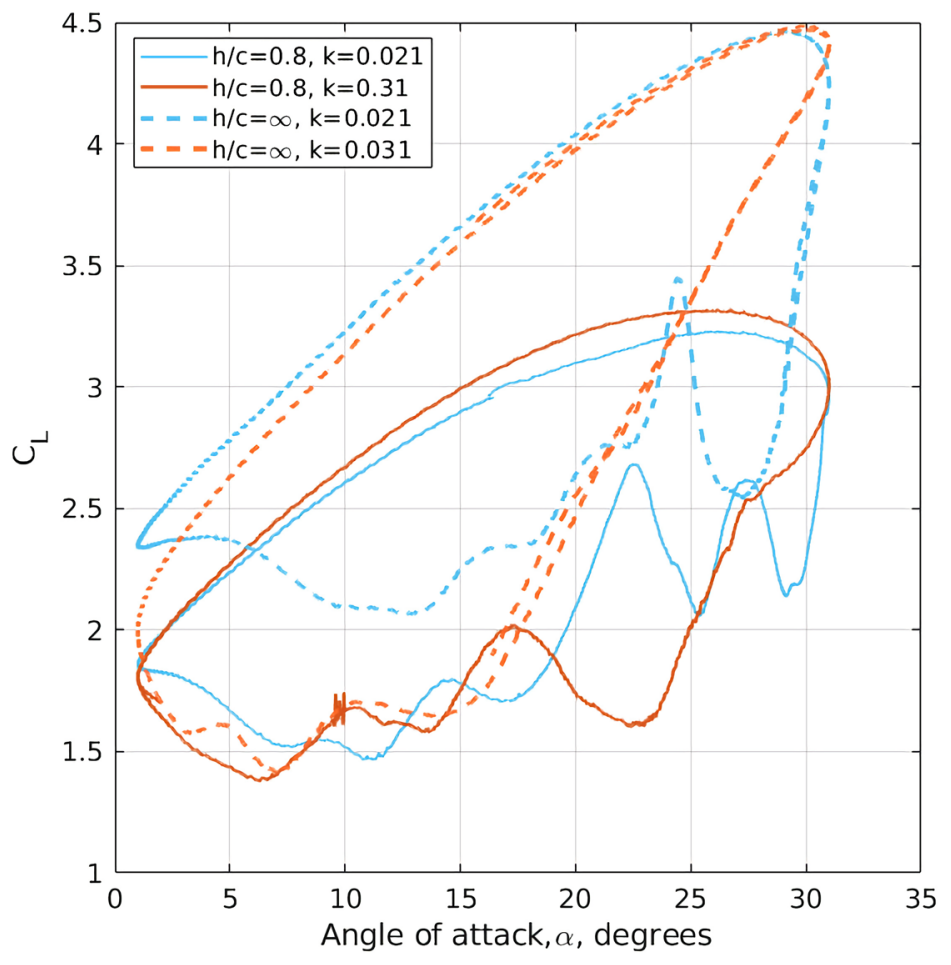

Figure 11. Comparison of lift force coefficient, $C_{L}$, for dynamic stall hysteresis loops at Reynolds number, $R e=5 \times 10^{6}$, for $30 \mathrm{P} 30 \mathrm{~N}$ airfoil with $h / c=\infty$ and $h / c=0.8$.

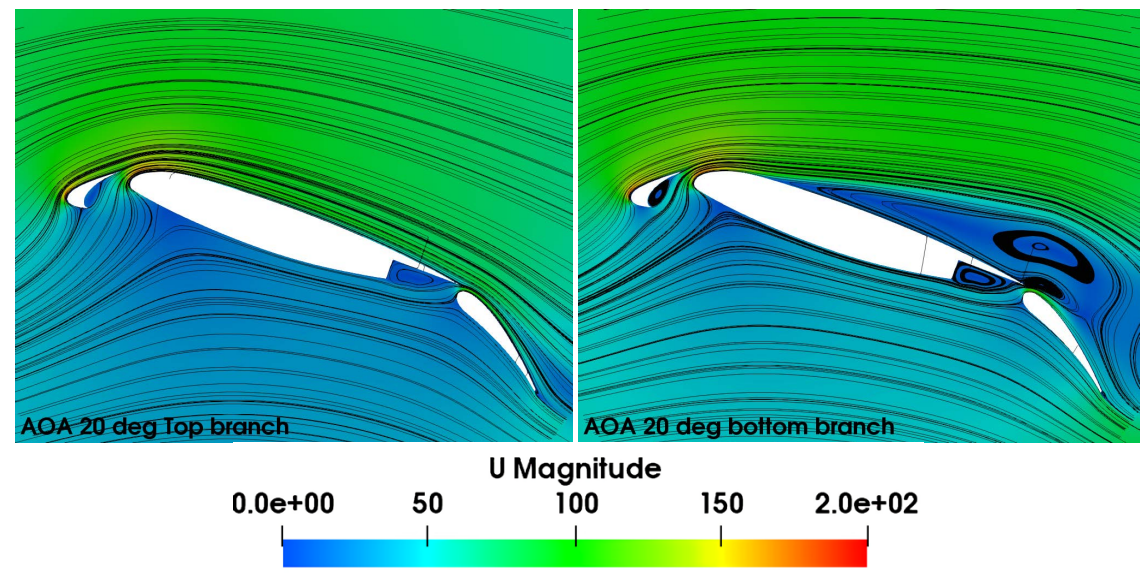

Figure 12. Streamlines of velocity for dynamic stall conditions at reduced frequency of $k=0.021$ and angle of attack, $\alpha=20^{\circ}$, top branch (left) and bottom branch (right).
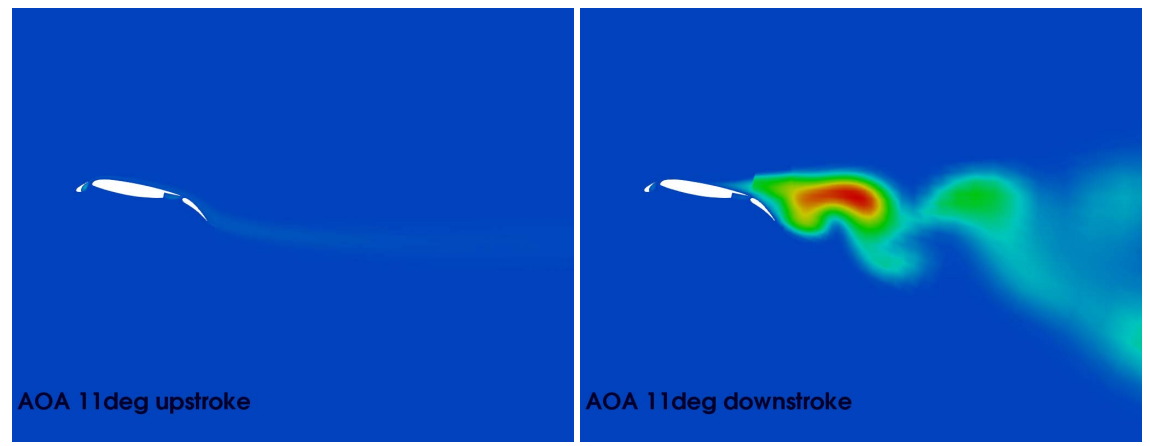


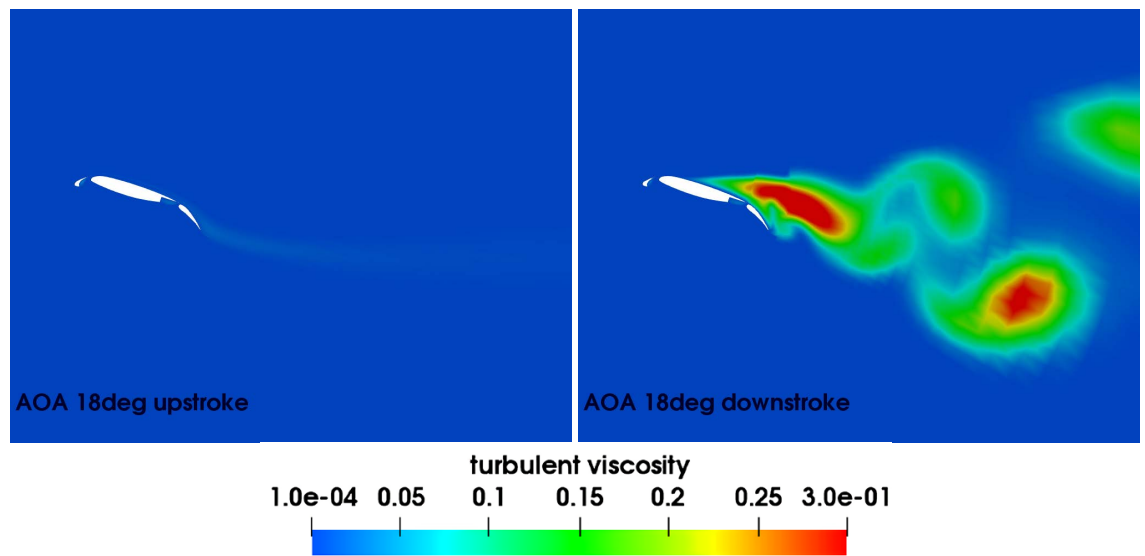

Figure 13. Contours of turbulent viscosity for dynamic stall conditions at reduced frequency of $k=0.021$ and angle of attack, $\alpha=11$ and $18.0^{\circ}$, during the upstroke and downstroke motion.

\section{Conclusion}

Stall aerodynamics of the three-element airfoil 30P30N has been investigated through CFD simulations using the OpenFOAM software both in free air and in close proximity to the ground at high Reynolds number $R e=5 \times 10^{6}$ and low Mach number $M=0.2$. The simulation results in static conditions show that the ground effect causes the significant reduction in the lift coefficient $C_{L}$ $\left(\Delta C_{L_{\max }}=-1.2\right)$. This unusual effect on the lift force due to the ground proximity is likely associated with the influence of a slotted flap on the pressure distribution of the airfoil [3] (Section 4.7). The validation of the CFD simulation results was done for static points only in free air conditions because of the absence of experimental data in close proximity to the ground. Dynamic stall simulations show an additional decrease in the lift coefficient $C_{L}(t)$ of the $30 \mathrm{P} 30 \mathrm{~N}$ airfoil during the downstroke both in free air conditions and in close proximity to the ground due to the development of a fully separated flow, delayed to lower angles of attack. This loss of lift requires special attention from a flight safety point of view, as dynamic stalls can occur during takeoff and landing due to pilot error or gusts of wind. An investigation of the finite aspect ratio wing with the high-lift $30 \mathrm{P} 30 \mathrm{~N}$ airfoil in free air and in the ground proximity is considered by the authors in the future.

\section{Conflicts of Interest}

The authors declare no conflicts of interest regarding the publication of this paper.

\section{References}

[1] Fang, L. (2011) Surpression of Stall on High-Camber Flap Using Upper-Surface Blowing. Technical Report Thesis, Cranfield University, Bedford.

[2] Klausmeyer, S.M. and Lin, J.C. (1997) Comparative Results from a CFD Challenge over a 2D Three-Element High-Lift Airfoil. Technical Report Technical Memorandum 112858, NASA, Washington DC.

https://ntrs.nasa.gov/api/citations/19970027380/downloads/19970027380.pdf 
[3] Hoak, D.E. (1960) The USAF Stability and Control DATCOM. Technical Report TR-83-3048, Air Force Wright Aeronautical Laboratories, Ohio, USA.

[4] Schroeder, J.A. (2012) Research and Technology in Support of Upset Prevention and Recovery Training. Technical Report AIAA Paper 2012-4567, AIAA Modeling and Simulation Technologies Conference, Minneapolis, 13-16 August 2012. https://doi.org/10.2514/6.2012-4567

[5] Belcastro, C.M. and Jacobson, S.R. (2010) Future Integrated Systems Concept for Preventing Aircraft Loss-of-Control Accidents. Technical Report AIAA Paper 2010-8142, Guidance, Navigation, and Control Conference, Toronto, 2-5 August 2010.

[6] Pascioni, J.A., Cattafesta, N.L. and Choudhari, M.M. (2014) An Experimental Investigation of the 30P30N Multi-Element High-Lift Airfoil. Technical Report AIAA 2014-3062, 20th AIAA/CEAS Aeroacoustics Conference, Atlanta, 16-20 June 2014. https://doi.org/10.2514/6.2014-3062

[7] Foundation, F.S. (2000) Approach-and-Landing Accident Reduction (ALAR) Briefing Note: 8.7-Crosswind Landing. Technical Report, Flight Safety Digest.

[8] Ahmed, M., Takasaki, T. and Kohama, Y. (2007) Aerodynamics of a NACA 4412 Airfoil in Ground Effect. AIAA Journal, 45, 37-47. https://doi.org/10.2514/1.23872

[9] Mahon, S. and Zhang, X. (2005) Computational Analysis of Pressure and Wake Characteristics of an Aerofoil in Ground Effect. Journal of Fluids Engineering, 127, 290-298. https://doi.org/10.1115/1.1891152

[10] Doig, G. and Barber, T. (2011) Considerations for Numerical Modeling of Inverted Wings in Ground Effect. AIAA Journal, 49, 2330-2333. https://doi.org/10.2514/1.J051273

[11] Zhang, X. and Zerihan, J. (2011) Edge Vortices of a Double-Element Wing in Ground Effect. Journal of Aircraft, 41, 1127-1137. https://doi.org/10.2514/1.1380

[12] Sereez, M., Abramov, N. and Goman, M.G. (2018) Impact of Ground Effect on Lateral Directional Stability during Take-Off and Landing. Open Journal of Fluid Dynamics, 8, 1-14. https://doi.org/10.4236/ojfd.2018.81001

[13] Mittal, S. and Saxena, P. (2000) Prediction of Hysteresis Associated with Static Stall of an Airfoil. AIAA Journal, 38, 2179-2189. https://doi.org/10.2514/2.1051

[14] Mittal, S. and Saxena, P. (2002) Hysteresis in Flow past a NACA 0012 Airfoil. Computer Methods in Applied Mechanics and Engineering, 191, 2207-2217. https://doi.org/10.1016/S0045-7825(01)00382-6

[15] Wang, S., Ingham, D.B., Ma, L., Pirkashanian, M. and Tao, Z. (2010) Numerical Investigations on Dynamic Stall of Low Reynolds Number Flowa Round Oscillating Airfoils. Computers and Fluids, 39, 1529-1541. https://doi.org/10.1016/j.compfluid.2010.05.004

[16] Wernert, P., Geissler, W., Raffel, M. and Kompenhas, J. (1996) Experimental and Numerical Investigations of Dynamic Stall on a Pitching Airfoil. AIAA Journal, 34, 982-989. https://doi.org/10.2514/3.13177

[17] Cummings, R.M. and Goreyshir, M. (2013) Challenges in the Aerodynamic Modelling of an Oscillating and Translating Airfoil at Large Incidence Angles. Aerospace Science and Technology, 28, 176-190. https://doi.org/10.1016/j.ast.2012.10.013

[18] Ekaterinaris, J.A. and Platzer, M.F. (1997) Computational Prediction of Airfoil Dynamic Stall. Progress in Aerospace Sciences, 33, 759-846. https://doi.org/10.1016/S0376-0421(97)00012-2

[19] Sereez, M., Abramov, N.B. and Goman, M.G. (2016) Computational Simulation of Airfoils Stall Aerodynamics at Low Reynolds Numbers. Technical Report Applied 
Aerodynamics Conference, RaES.

[20] Berg, B.V.D. (1981) Role of Laminar Bubbles in Airfoil Leading-Edge Stalls. AIAA, 19, 553-570. https://doi.org/10.2514/3.7798

[21] Visbal, M.R. and Garmann, D.J. (2018) Analysis of Dynamic Stall on Pitching Airfoil Using High-Fidelity Large-Eddy Simulations. AIAA Journal, 56, 46-63. https://doi.org/10.2514/1.J056108

[22] Esfahani, A., Webb, N. and Samimy, M. (2019) Flow Separation Control over a Thin Post-Stall Airfoil: Effects of Excitation Frequency. AIAA Journal, 57, 1826-1837. https://doi.org/10.2514/1.J057796

[23] Sereez, M., Abramov, N. and Goman, M.G. (2021) Prediction of Static Aerodynamic Hysteresis on a Thin Airfoil Using OpenFOAM. Journal of Aircraft, 58, 374-382. https://doi.org/10.2514/1.C035956

[24] Williams, D.R., Reißner, F., Greenblatt, D., Müller-Vahl, H. and Strangfeld, C. (2017) Modeling Lift Hysteresis on Pitching Airfoils with a Modified Goman-Khrabrov Model. AIAA Journal, 55, 403-409. https://doi.org/10.2514/1.J054937

[25] Luchtenburg, D.M., Rowley, W.C., Lohry, M.W., Martinelli, L. and Stengel, R.F. (2015) Unsteady High-Angle-of-Attack Aerodynamic Models of a Generic Jet Transport. Journal of Aircraft, 52, 890-895. https://doi.org/10.2514/1.C032976

[26] Abramov, N.B., Goman, M.G., Khrabrov, A.N. and Soemarwoto, B.I. (2019) Aerodynamic Modeling for Poststall Flight Simulation of a Transport Airplane. Journal of Aircraft, 56, 1427-1440. https://doi.org/10.2514/1.C034790

[27] OpenFOAM (2021) OpenFOAM: The Open Source Computational Fluid Dynamics Toolbox. http://www.openfoam.com

[28] ANSYS (2021) Fluent: Fluid Simulations Software. https://www.ansys.com/en-gb/products/fluids/ansys-fluent

[29] Spalart, P.R. and Allmaras, S.R. (1992) A One-Equation Turbulence Model for Aerodynamic Flows. Technical Report, 30 th Aerospace Sciences Meeting and Exhibit, Reno, 6-9 January 1992. https://doi.org/10.2514/6.1992-439

[30] Menter, F.R. (1994) Two-Equation Eddy-Viscosity Turbulence Models for Engineering Applications. AIAA Journal, 32, 1598-1605. https://doi.org/10.2514/3.12149

[31] Jameson, A. (1991) Time Dependent Calculations Using Multigrid, with Applications to Unsteady Flows past Airfoils and Wings. Technical Report, 10 th Computational Fluid Dynamics Conference, Honolulu, 24-26 June 1991. https://doi.org/10.2514/6.1991-1596

[32] Steinbach, D. and Jacob, K. (1991) Some Aerodynamic Aspects of Wings near Ground. Transactions of the Japan Society for Aero-Nautical and Space Sciences, 34, 56-70.

[33] Hoak, D.E. and Finck, R.D. (1974) USAF Stability and DAR 303. Technical Report, A2-1 to A2-12, Flight Safety Digest.

[34] Qin, X.G., Liu, P.Q. and Qu, Q.L. (2009) Aerodynamics of a Multi-Element Airfoil near Ground. Tsinghua Science and Technology, 14, 94-99. https://doi.org/10.1016/S1007-0214(10)70039-0

[35] Partha, M. and Balakrishnan, N. (2014) Discrete Vortex Method-Based Model for Ground-Effect Studies. AIAA Journal, 52, 2817-2828. https://doi.org/10.2514/1.J052920

[36] Kim, S., Alonso, J.J. and Jameson, A. (2004) Multi-Element High-Lift Configuration Design Optimization Using Viscous Continuous Adjoint Method. Journal of Aircraft, 41, 1082-1096. https://doi.org/10.2514/1.17 


\section{Nomenclature}

$C_{y}=$ normal force coefficient

$C_{L}=$ lift coefficient

$C_{M}=$ pitching moment coefficient

$c, S_{\text {ref }}=$ reference chord length and area

$C_{L \max }=$ maximum lift coefficient

$U_{\text {ref }}=$ reference flow velocity

$k=$ reduced frequency, $\omega c /\left(2 U_{\text {ref }}\right)$

$R e=$ Reynolds number

$t=$ physical time

$\lambda=$ aspect ratio

$\alpha=$ angle of attack

$\alpha_{m}=$ mean angle of attack

$\alpha_{a}=$ amplitude of angle of attack variation

$h / c=$ height to chord ratio 